\title{
Investigation of the Quaternion Dynamical System
}

\author{
Yasmin Omar \\ Department of Mathematics, Faculty of Science, Damietta University, Damietta, Egypt \\ Email: yasminomar@du.edu.eg, yasmin.omar985@gmail.com
}

How to cite this paper: Omar, Y. (2017) Investigation of the Quaternion Dynamical System. Journal of Applied Mathematics and Physics, 5, 131-136.

http://dx.doi.org/10.4236/jamp.2017.51014

Received: November 30, 2016

Accepted: January 22, 2017

Published: January 25, 2017

Copyright $\odot 2017$ by author and Scientific Research Publishing Inc. This work is licensed under the Creative Commons Attribution International License (CC BY 4.0).

http://creativecommons.org/licenses/by/4.0/

\begin{abstract}
The quaternion Mandelbrot set is one of the most important sets in mathematics. In this paper we first give some properties of the quaternion algebra. Then, we introduce the quternion dynamical system. We are concerned with analytical and numerical investigation of the quaternion dynamical system.
\end{abstract}

\section{Keywords}

Quaternion Algebra, Mandelbrot Set, Qaternion Dynamical System, Equilibrium Points; Stability, Lyapunov Exponents

\section{Introduction}

The well-known concept of quaternions was introduced by Hamilton in 1866 [1]. Quaternions are the generalization of complex numbers. A quaternion has four components, i.e., one real part and three imaginary parts:

$$
q=q_{r}+q_{i} i+q_{j} j+q_{k} k,
$$

where $i, j, k$ obey the rules as below:

$$
i j=j i=k, j k=k j=i, k i=i k=j, i^{2}=j^{2}=k^{2}=1 .
$$

Every quaternion $q$ has its conjugate, $q^{*}=q_{r}-q_{i} i-q_{j} j-q_{k} k$. From (1), we can find that the multiplication rule of quaternions is not commutative.

Quaternion algebra is an excellent tool for the treatment of three- and fourdimensional rotations. Therefore studying quaternion is an important task. Moreover it plays an important role in areas of computer graphics, biomechanics, molecular modeling and mesh deformation.

The topic of Mandelbrot dynamical system [2] was studied by many authors [3] [4] [5], but still interesting. In fact, we found that there is an strong relation between the well known Mandelbrot dynamical system and the new one, the so called quaternion dynamical system. 
In the following sections, we give an overview of Mandelbrot dynamical system and study quaternion dynamical system.

\section{Mandelbort Dynamical System}

The Mandelbrot set is a mathematical set of points whose boundary is a distinctive and easily recognizable two-dimensional fractal shape. Images of the Mandelbrot set are made by sampling complex numbers and determining for each whether the result tends towards infinity when a particular mathematical operation is iterated on it. More precisely, the Mandelbrot set is the set of values of $C$ in the complex plane for which the orbit of 0 under iteration of the complex quadratic polynomial

$$
z_{n+1}=z_{n}^{2}+C
$$

remains bounded. That is, a complex number $C$ is part of the Mandelbrot set if, when starting with $z_{0}=0$ and applying the iteration repeatedly, the absolute value of remains bounded however large $n$ gets. More Precisely given this beginning value $z_{0}=0$, and a value for $C$, one can generate $z_{1}$. Then, using this value of $z_{1}$, one can generate $z_{2}$, and so on. The Equation (2) in itself is very simple but the resulting graph is so complicated. A computer tool is the most powerful tool we have to work with this system due to its fast computational capabilities. This dynamical system was studied by many other authors, see e.g. [2] [6].

\section{Quaternions}

This section is concerned with some algebraic properties of the non-commutative division quaternion algebra. Let $\mathbb{R}$ and $\mathbb{C}$ denote the field of the real and complex numbers respectively. Let $\mathbb{Q}$ be a four-dimensional real algebra generated by the identity element $e$ and the symbols $i, j$ and $k$. So

$$
\mathbb{Q}=\left\{x_{o} e+x_{1} i+x_{2} j+x_{3} k: x_{o}, \cdots, x_{3} \in \mathbb{R}\right\} .
$$

Quaternions are added together component by component, and quaternion multiplication is given by the quaternion relations (Hamiltonian conditions), given in (1).

If $x$ and $y$ are any (real) scalars, while $u, v$ are any two of $e, i, j, k$, then the product $(a u)(b v)$ is defined as $(a b)(u v)$, see [5].

\section{Some Properties of Quaternion Algebra $\mathbb{Q}$}

(A) For any $x=x_{o}+x_{1} i+x_{2} j+x_{3} k \in \mathbb{Q}$, we define

1. The real part of $x, \operatorname{Re} x=x_{0}$.

2. The complex part of $x$, Co $x=x_{1}+x_{2} i$.

3. The imaginary part of $x, \operatorname{Im} x=x_{1} i+x_{2} j+x_{3} k$.

4. The conjugate of $x, \bar{x}=x^{*}=x_{o}-x_{1} i-x_{2} j-x_{3} k$.

5. The norm of $x,|x|=\sqrt{x x^{*}}=\sqrt{x^{*} x}=\sqrt{x_{0}^{2}+x_{1}^{2}+x_{2}^{2}+x_{3}^{2}}$. And $x$ is said to be a unit quaternion if its norm is 1 . Hence we can see that each non zero qua- 
ternion $x$ has inverse $x^{-1}=\frac{1}{|x|}$. Then the algebra $\mathbb{Q}$ is a division algebra.

6. The product of two quaternions $x, y$ is

$$
\begin{aligned}
x y= & \left(x_{o}+x_{1} i+x_{2} j+x_{3} k\right)\left(y_{o}+y_{1} i+y_{2} j+x_{3} k\right) \\
= & \left(x_{o} y_{o}-x_{1} y_{1}-x_{2} y_{2}-x_{3} y_{3}\right)+\left(x_{o} y_{1}+x_{1} y_{o}+x_{2} y_{3}-x_{3} y_{2}\right) i \\
& +\left(x_{o} y_{2}-x_{1} y_{3}+x_{2} y_{o}+x_{3} y_{1}\right) j+\left(x_{o} y_{3}+x_{1} y_{2}-x_{2} y_{1}+x_{3} y_{o}\right) k
\end{aligned}
$$

Note that $x y \neq y x$. Hence $\mathbb{Q}$ is a non-commutative.

(B) Every quaternion $q$ can be uniquely expressed as $q=z_{1}+z_{2} j$, where $z_{1}$ and $z_{2}$ are complex numbers by the maping

$$
z_{1}+z_{2} j \rightarrow\left(\begin{array}{cc}
Z_{1} & Z_{2} \\
-\bar{Z}_{2} & \bar{Z}_{1}
\end{array}\right)
$$

(C) Two quaternions $x$ and $y$ are said to be similar if there exists a nonzero quaternion $v$ such that $v^{-1} x v=y$, this is written as $x \sim y$. In other words $x$ and $y$ are similar if and only if there is a unit quaternion $v$ such that $v^{-1} x v=y$, and two similar quaternions have the same norm. It is easy to check that $\sim$ is an equivalence relation on the quaternions. We represent the equivalence class containing $x$ by $[x]$.

Remark 1. $\mathbb{Q}$ is a real division algebra: $|p q|=|p||q|$ for all $p, q \in \mathbb{Q}$.

\section{Quaternion Dynamical System}

In this section, we study a new dynamical system, the so called quaternion dynamical system. Define the following quaternion dynamical system

$$
Q_{n+1}=Q_{n}^{2}+C, C \text { is quaternion constant. }
$$

Proposition 1. The orbit of system (3) is either bounded by $|C|+1$ or unbounded.

Proof.

$$
\left|Q_{n+1}\right|=\left|Q_{n}^{2}+C\right| \geq\left|Q_{n}\right|^{2}-|C|
$$

If $\left|Q_{n}\right| \geq|C|+1$, then

$$
\begin{gathered}
\left|Q_{n+1}\right| \geq|C|^{2}+|C|+1 . \\
\left|Q_{n+2}\right| \geq 3|C|^{2}+|C|+1 .
\end{gathered}
$$

Mathematical induction of $n$ gives

$$
\left|Q_{n+k}\right| \geq\left(2^{k}-1\right)|C|^{2}+|C|+1
$$

Hence we conclude that $\left|Q_{n+k}\right|$ is bounded.

Definition 1. Quaternion Mandelbrot set is

$$
\left\{C / Q_{n+1}^{C} \text { is bounded } \forall n=1,2, \cdots\right\}
$$

Proposition 2. If $|Q| \geq|c|,|Q|>|2|$, then the trajectory generated by (3) is unbounded. Hence quaternion Mandelbrot set is bounded by $|C|=2$.

Proof. Let $\left|Q_{n}\right|>2,\left|Q_{n}\right| \geq|C|$. Then 


$$
\begin{aligned}
\left|Q_{n+1}\right| & =\left|Q_{n}^{2}+C\right| \geq\left|Q_{n}\right|^{2}-|C|\left(\left|Q_{n}\right| \geq|C|\right) \\
& \geq\left|Q_{n}\right|^{2}-\left|Q_{n}\right|=\left|Q_{n}\right|\left(\left|Q_{n}\right|-1\right) .
\end{aligned}
$$

This implies that $\left|Q_{n+1}\right|>\left|Q_{n}\right|>2$. Hence the trajectory is unbounded.

Since $Q_{1}(0)=C$, then $\left|Q_{1}(0)\right|=|C|>2$. Using part one, hence $Q_{n}(0)$ is bounded. This completes the proof of the proposition.

\section{The Equilibrium Point and Its Stability}

In this section we use the $2 \times 2$ Pauli matrices approach, in which a quaternion can be written in matrix form as:

$$
Q=X I+i \underline{\sigma} \cdot \underline{W},
$$

where, $I=\left(\begin{array}{ll}1 & 0 \\ 0 & 1\end{array}\right)$ is the $2 \times 2$ unit matrix, $\underline{\sigma}=\left(\sigma_{1}, \sigma_{2}, \sigma_{3}\right)$,

$$
\sigma_{1}=\left(\begin{array}{ll}
0 & 1 \\
1 & 0
\end{array}\right), \sigma_{2}=\left(\begin{array}{cc}
0 & -i \\
i & 0
\end{array}\right), \sigma_{3}=\left(\begin{array}{cc}
1 & 0 \\
0 & -1
\end{array}\right)
$$

are the Pauli $2 \times 2$ matrices and the vector $\underline{W}=(Y, U, V)$. The quaternion components $(X, \underline{W}) \in \mathbb{R}^{4}$.

Following the analogy of the complex quadratic map [7], we introduce the quaternionic quadratic map

$$
Q_{n+1}=Q_{n}^{2}+C,
$$

where

$$
C=c_{0} I+i \underline{\sigma} \cdot \underline{K}
$$

with

$$
\underline{K}=\left(c_{1}, c_{2}, c_{3}\right) \quad \text { and } \quad\left\{c_{0}, \underline{K}\right\} \in \mathbb{R}^{4}
$$

The corresponding system of (4) in terms $X, \underline{W}_{n}$ is

$$
\begin{aligned}
X_{n+1} & =X_{n}^{2}-Y_{n}^{2}-U_{n}^{2}-V_{n}^{2}+c_{0}, \\
Y_{n+1} & =2 X_{n} Y_{n}+c_{1}, \\
U_{n+1} & =2 X_{n} U_{n}+c_{2}, \\
V_{n+1} & =2 X_{n} V_{n}+c_{3} .
\end{aligned}
$$

The equilibrium point for Equation (4) is defined by the equation

$$
Q=Q^{2}+C \text {. }
$$

The solution of equilibrium is given by

$$
Q=\frac{1 \pm \sqrt{1-4 C}}{2},
$$

and it is stable if all the eigenvalues $\lambda$ of the matrix $\boldsymbol{J}$, where

$$
q_{n+1}=\left(\begin{array}{cccc}
2 X & -2 Y & -2 U & -2 V \\
2 Y & 2 X & 0 & 0 \\
2 U & 0 & 2 X & 0 \\
2 V & 0 & 0 & 2 X
\end{array}\right) q_{n}=J q_{n}
$$

satisfy $|\lambda|<1$. 
In terms of $X$ and $\underline{W}$, the equilibrium point is stable if and only if

$$
X^{2}+\underline{W}^{2}<\frac{1}{4}
$$

The numerical simulation in Figure 1 carried out with MATLAB serve for illustration the quaternion dynamical system (3) in terms of $X, Y, U$ for the three different values of $C$.

\section{Lyapunov Exponents}

In this section we introduce a quantitative measure of chaos, the so called Lyapunov exponents [8] [9]. This quantitative measure of chaos is so important for several reasons. The most important reason is that, it allows us to define exactly what the meaning of chaos is. More precisely, in order to analyze the parameters influence in periodic and complex behavior, we compute the maximal Lyapunov exponents. The maximal Lyapunov exponent has one of the following cases.

(1) negative (stable equilibrium point),

(2) zero (bifurcation point),

(3) positive (chaos).

In order to study the relations between the local stability of the equilibrium point for the quaternion dynamical system (3) and the parameters $C$ based on maximal Lyapunov exponents, Figure 2 illustrates the related maximal Lyapunov exponents. A positive value of maximal Lyapunov exponents implies sensitive dependence on initial condition for chaotic behavior. From the maximal Lyapunov exponents figure, it is easy to determine the parameter sets for which
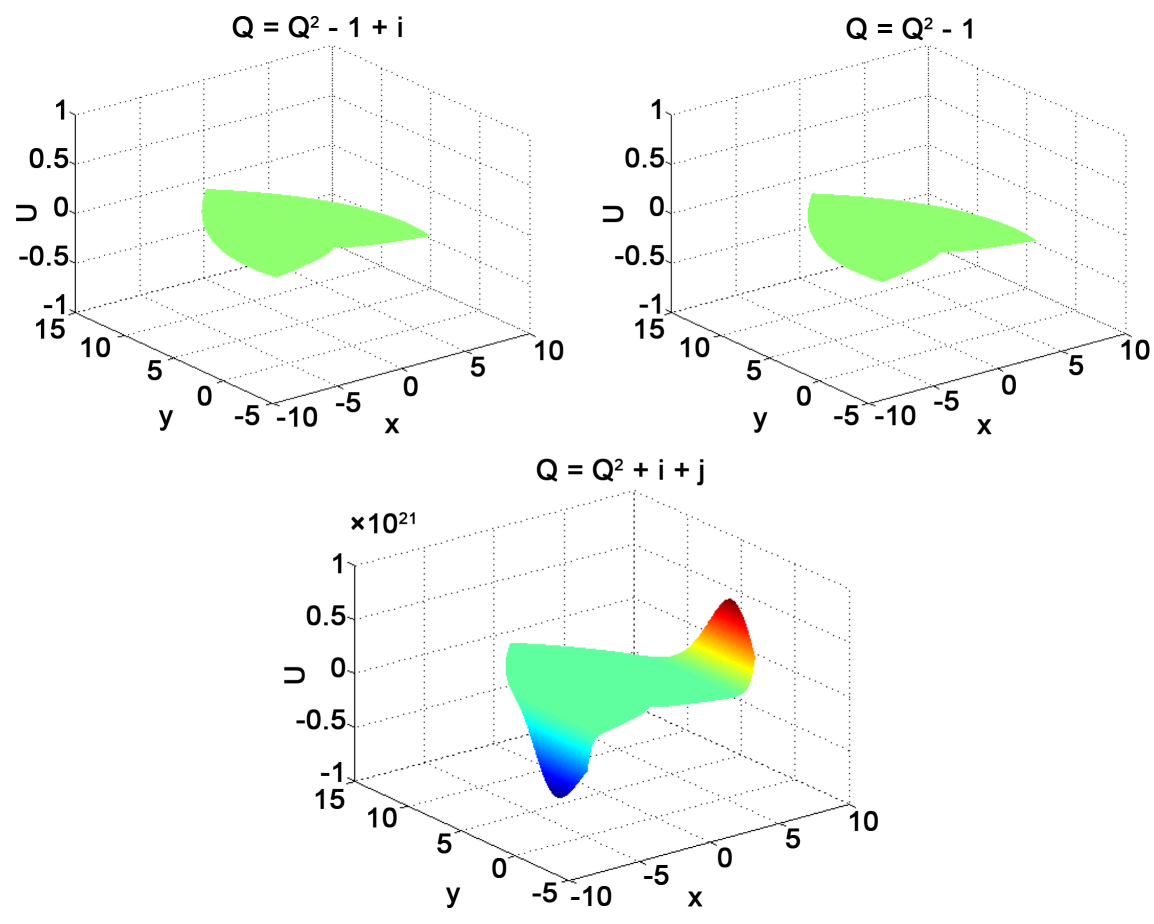

Figure 1. The quaternion dynamical system. 

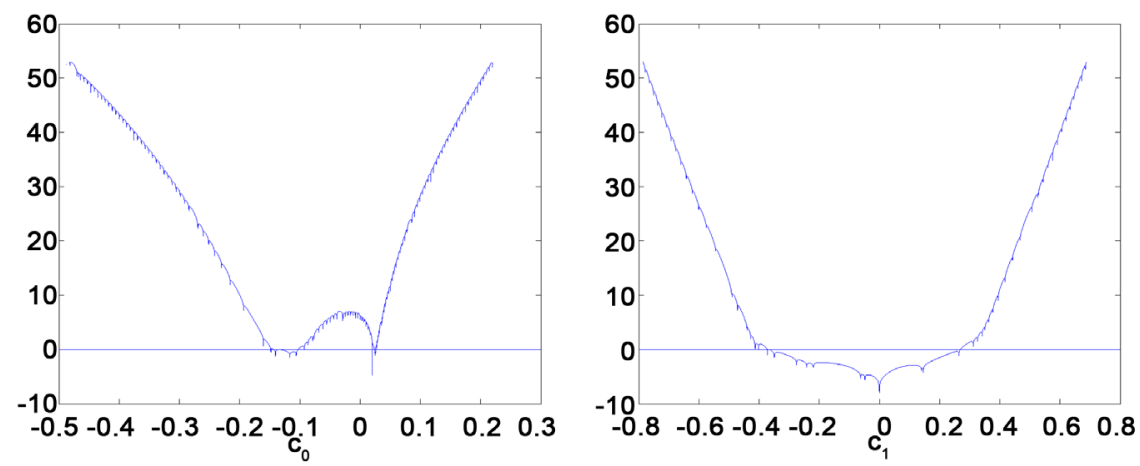

Figure 2. The maximal Lyapunov exponent with respect to $c_{0}$ and $c_{1}$.

the system converges to cycles and chaotic behavior.

Here we give the Lyapunov exponents for the quaternion dynamical system (3).

\section{References}

[1] Hamilton, W.R. (1866) Elements of Quaternions. Longmans, Green and Co., London.

[2] Mandelbrot, B. (1982) The Fractal Geometry of Nature. Freeman, San Francisco.

[3] Gomatam, J., Doyle, J., Steves, B. and Mcfarlane, I. (1995) Generalization of the Mandelbrot Set: Quaternionic Quadratic Maps. Chaos, Solitonr and Fractals, 5, 971 986. https://doi.org/10.1016/0960-0779(94)00163-K

[4] Norton, A. (1989) Julia Sets in the Quaternions. Computers and Graphics, 13, 266278. https://doi.org/10.1016/0097-8493(89)90071-X

[5] Zhang, F. (1997) Quaternions and Matrices of Quaternions. Linear Algebra and Its Applications, 251, 21-57. https://doi.org/10.1016/0024-3795(95)00543-9

[6] Klebaoff, A. (2001) Pi in the Mandelbrot Set. Fractals, 9, 393-402. https://doi.org/10.1142/S0218348X01000828

[7] Mandelbrot, B. (1980) Fractal Aspects of the Iteration of $z \rightarrow \lambda z(1-z)$ for Complex $\lambda$ and $Z$. Annals of the New York Academy of Sciences, 357, 249-259. https://doi.org/10.1111/j.1749-6632.1980.tb29690.x

[8] Eckmann, J.-P. and Ruelle, D. (1992) Fundamental Limitations for Estimating Dimensions and Lyapunov Exponents in Dynamical Systems. Physica D, 56, 185-187. https://doi.org/10.1016/0167-2789(92)90023-G

[9] Gencay, R. and Davis Dechert, W. (1992) An Algorithm for the n Lyapunov Exponents of an n-Dimensional Unknown Dynamical System. Physica D, 59, 142-157. https://doi.org/10.1016/0167-2789(92)90210-E 
Submit or recommend next manuscript to SCIRP and we will provide best service for you:

Accepting pre-submission inquiries through Email, Facebook, LinkedIn, Twitter, etc. A wide selection of journals (inclusive of 9 subjects, more than 200 journals)

Providing 24-hour high-quality service

User-friendly online submission system

Fair and swift peer-review system

Efficient typesetting and proofreading procedure

Display of the result of downloads and visits, as well as the number of cited articles Maximum dissemination of your research work

Submit your manuscript at: http://papersubmission.scirp.org/

Or contact jamp@scirp.org 